\title{
Schizophrenia and Obsessive-Compulsive Disorder: Are They Related Disorders?
}

"Older nosologic

schemes in the field

of neuropsychiatry

regarded

schizophrenia and

obsessive-compulsive

disorder as mutually

exclusive disorders,

completely separate

and unrelated,

with no coexistence

between them.

Such categorical

dogmatism is

curious considering

that this "rule"

was totally

unfounded

by empirical

observation."

Dr. McGlashan is executive director, Yale Psychiatric Institute and professor of psychiatry, Yale University School of Medicine.

\section{INTRLDUCTION}

Older nosologic schemes in the field of neuropsychiatry regarded schizophrenia (SCZ) and obsessive-compulsive disorder (OCD) as mutually exclusive disorders, completely separate and unrelated, with no coexistence between them. Such categorical dogmatism is curious considering that this "rule" was totally unfounded by empirical observation. However, the rule was gradually ignored, and data emerged that contradicted mutual exclusivity and introduced much uncertainty and confusion to the heretofore neat and orderly picture of SCZ and OCD as separate entities.

This article reviews the papers presented in the March and April issues of CNS Spectrums that represent an initial look at the co-occurance and interaction between SCZ and OCD at the levels of description and treatment response. All of them prove, by the examples described herein, that these two disorders are not mutually exclusive. In fact, the data they present suggest that SCZ and OCD may be related, and they offer clues as to the nature of that linkage. The data presented in this collection of papers are very preliminary and almost entirely descriptive in nature. Yet they are compelling and provocative, suggesting that the study and treatment of comorbid SCZ and OCD will yield much in the future about the nature of these disorders, their interaction, and their treatment.

HYPDTHESIZING THE

RELATIONSHIP DF

SCHIZLPHRENIA AND

\section{DESESSIVE-CDMPULSIVE}

\section{DISORDER}

In their profile of obsessive-compulsive (OC) symptoms in schizophrenia, Porto and colleagues ${ }^{1}$ present a comprehensive assessment of OC symptoms in a sample of 50 chronic schizophrenic outpatients. OC symptoms were present in $46 \%$ of the sample, and OCD was present in $26 \%$, which shows considerable overlap indeed, especially if the sample was not preselected for OC features. Where overlap occurred, there appeared to be three patterns. In the first pattern, the OCD symptoms appeared unrelated to the psychotic symptoms. In the second, the OC signs and symptoms appeared related, but not restricted, to the psychotic signs and symptoms. In the third, the OC symptoms appeared to be on a continuum with psychosis in so far as obsessions would become delusions during more active phases and return to obsessions (with insight) during remissions. While the work of this group is descriptive at the symptomatic level, their phenomenologic groupings may be extrapolated into the following alternate hypotheses about the relationship between SCZ and OCD as disorders: (1) SCZ and $O C D$ are separate entities that can cooccur; (2) SCZ and OCD are one disorder and represent different aspects of a continuum; and (3) SCZ and OCD are different disorders with shared elements of psychopathology and symptom pathophysiology.

\section{WHAT EVIDENCE DD THE PAPERS IN THESE ISSUES PRESENT FDR DR AGAINST} EACH HYPDTHESIS?

If, in their published literature, Sasson et $\mathrm{al}^{2}$ are correct in asserting that $15 \%$ of chronic schizophrenic patients also suffer from OCD, then our first hypothesis that SCZ and OCD are separate disorders must be invalid. Schizophrenia occurs at a prevalence of $1 \%$ in the general population and OCD at a prevalence of roughly $2 \%$. If they were independent categories, their rate of overlap would range between $1 \%$ and $2 \%$. Fifteen percent certainly suggests some kind of linkage. These data are provocative and clearly call for more clinical epidemiologic studies to test the frequency and comorbidity with rigor and reliability. Most reports to date have counted the frequency of OC symptoms and OCD in chronic schizophrenic samples. At least two types of clinical epidemiologic studies are needed: the rate of SCZ in OCD and the rate of OCD in SCZ. Furthermore, the latter studies should involve SCZ samples that are acutely, as well as chronically, disabled. 
Could SCZ and OCD be one entity? YaryuraTobias and colleagues ${ }^{3}$ address this question most directly by comparing patients with OCD and with SCZ on a variety of symptom profiles. They conclude that SCZ and OCD share symptoms and behaviors without losing nosological individuality. This suggests that the assumption of syndromal unity is incorrect. Failure to support this hypothesis probably would generate little criticism among workers in the field, especially clinicians who regard and usually treat these disorders as different. While overlap between the disorders is frequent, it is also clear that both SCZ without OCD and OCD without SCZ are common. If they were but different manifestations of the same entity, we could probably expect to see far greater comorbidity, as well as more confluence of one disorder into the other on the basis of acuteness and/or severity.

At the same time, the hypothesis of unity should not be dismissed. Many of the nonsymptomatic clinical parameters are similar between the disorders. Both tend to begin in adolescence and early adulthood, and the age of onset in both is earlier in males. The timing of onset in both can be variable, ie, gradual or acute, and the longitudinal course can fluctuate in severity depending upon ambient levels of stress. Episodic exacerbations and longitudinal deterioration can occur in both disorders. Finally, familial patterns are present in both disorders, ie, higher frequencies of disorder in first-degree relatives versus the general population, and higher frequencies in monozygotic twins versus dizygotic twins. None of these observations suggest identity, but they do imply that we should be cautious in rejecting such a possibility.

The last hypothesis is a hybrid of the first two: OCD and SCZ are different disorders that share elements of symptomatology, psychopathology, and pathophysiology. The data of Berman and colleagues ${ }^{4}$ support this view. They assessed 30 schizophrenic patients and found $25 \%$ to have significant oC symptoms. The $O C$ and non-OC schizophrenic patients were not different on the positive and negative symptoms of schizophrenia, suggesting independence of $O C$ and psychosis phenomenology. On the other hand, the schizophrenic patients with OC symptoms scored deviantly on several neuropsychological subtests that are typically abnormal in nonschizophrenic OC patients. These tests involve visual-spatial skills, delayed nonverbal memory, and cogni- tive shifting abilities. Berman and colleagues say nothing about how schizophrenic patients without OCD score on these subtests; however, given the well-known pervasive deficits across neuropsychological profiles among schizophrenic patients, especially on tests of executive functioning such as the Wisconsin Card Sorting Test, it is unlikely the deficits recorded here are unique to OCD psychopathology. A study comparing neuropsychological profiles among three groups, ie, SCZ without OCD, OCD without SCZ, and SCZ with OCD, should be the next step. In the meantime, the study by Berman et al suggests that while there is independence between OCD and SCZ at the symptom level (OC symptoms vs positive and negative psychotic symptoms), there may be less independence at the level of cognitive functioning.

The treatment response data are mixed and confusing. Sasson and colleagues ${ }^{2}$ found that open-label clomipramine reduced $O C$ symptoms and psychosis in a significant subset of 18 patients with comorbid OCD and SCZ. Bermanzohn et $\mathrm{al}^{5}$ reported cross reactivity of anti-obsessional agents on psychosis in refractory delusional states, arguing that

\section{PEDIATRIC NEUROLOGIST PARTNERSHIP OPPORTUNITY}


these might in actuality be OCD in psychotic proportion. Cross-reactivity of neuroleptics for OC symp-toms has recently been reported in the form of risperidone augmentation of SSRI treatment of refractory OCD. ${ }^{6}$ Overall, data suggest both specificity and cross-reactivity, but the evidence is very preliminary.

"Cross-reactivity of

neuroleptics for

OC symptoms has

recently been

reported in the

form of risperidone

augmentation of

SSRI treatment of

refractory $0 \mathrm{CD}$.

Overall, data suggest

both specificity and

cross-reactivity,

but the evidence is

very preliminary."

\section{REFERENCES}

1. Porto L, Bermanzohn PC, Pollack S, Morrissey R, Siris SG. A profile of obsessive-compulsive symptoms in schizophrenia. CNS Spectrums. 1997;3:321-25.

2. Sasson Y, Bermanzohn PC, Zohar J. Treatment of obsessivecompulsive (OC) syndromes in schizophrenia. CNS Spectrums. 1997:4:34-45.

3. Yaryura-Tobias JA, Stevens KP, Neziroglu F, Grunes MS. Obsessive-compulsive disorder and schizophrenia: a phenomenological perspective of shared pathology. CNS Spectrums. 1997;4:21-25.

4. Berman I. Pappas D, Berman SM. Obsessive-compulsive symptoms in schizophrenia: are they manifestations of a distinct subclass of schizophrenia? CNS Spectrums. 1997;3:34-36.

5. Bermanzohn PC, Porto L, Arlow PB, et al. Are some neuroleptic-refractory symptoms of schizophrenia really obsessions? CNS Spectrums. 1997;3:50-57.

6. Saxena S, Wang D, Bystritsky A, Baxter L. Risperidone augmentation of SRI treatment for refractory obsessive-compulsive disorder. J Clin Psychiatry, 1996;57:303-306.

7. MoGlashan TH. Borderline personality disorder and unipolar affective disorder: long-term effects of comorbidity. $J$ Nerv Ment Dis. 1987:175:467-473.

8. Fenton WS, McGlashan TH. The prognostic significance of obsessive-compulsive symptoms in schizophrenia. Am J Psychiatry. 1986;143:437-441.

9. Hoffman RE, Oates E, Hafner RJ, Hustig HH. McGlashan TH. Semantic organization of hallucinated "voices" in schizophrenia. Am J Psychiatry. 1994;151:1229-1230. 


\section{There's a new reason to smile...}

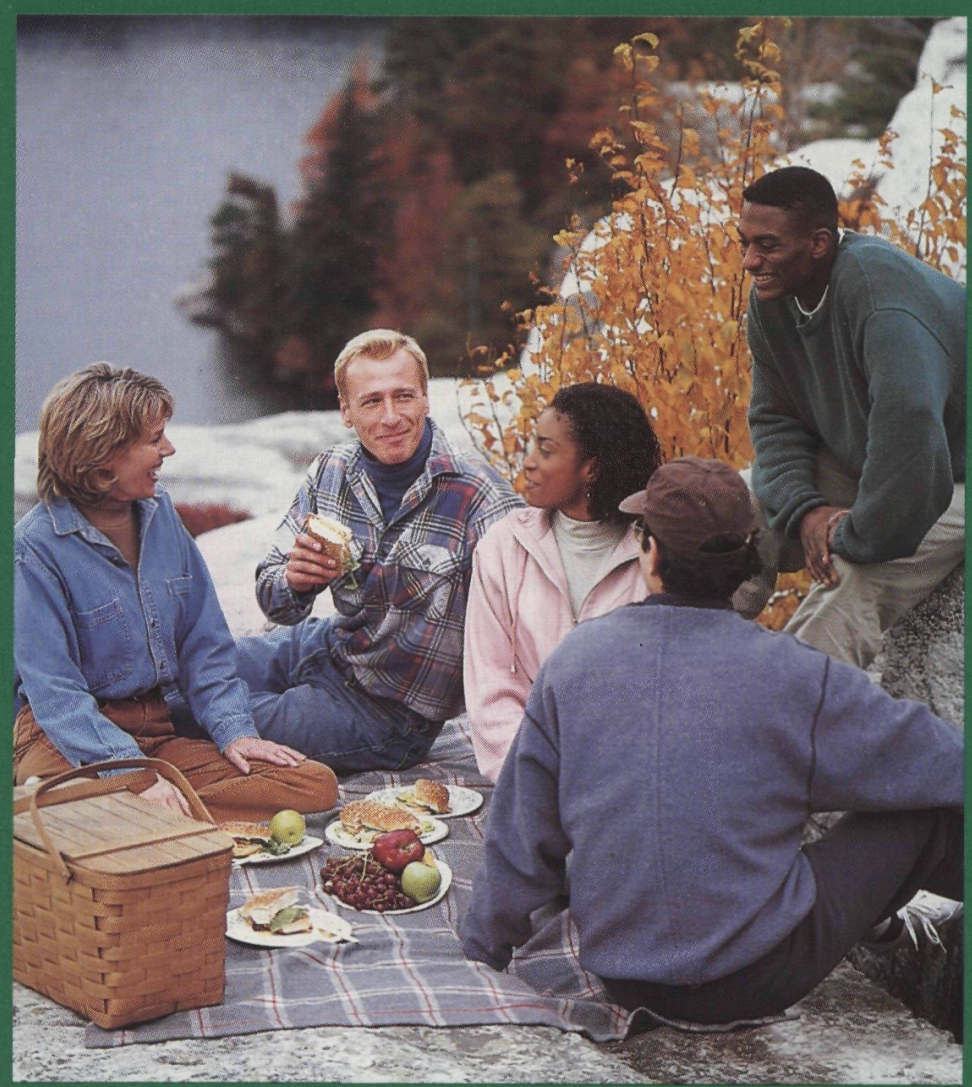

\section{Now aroailable for the treatment of Obsessive-Compulsive Disorder.}

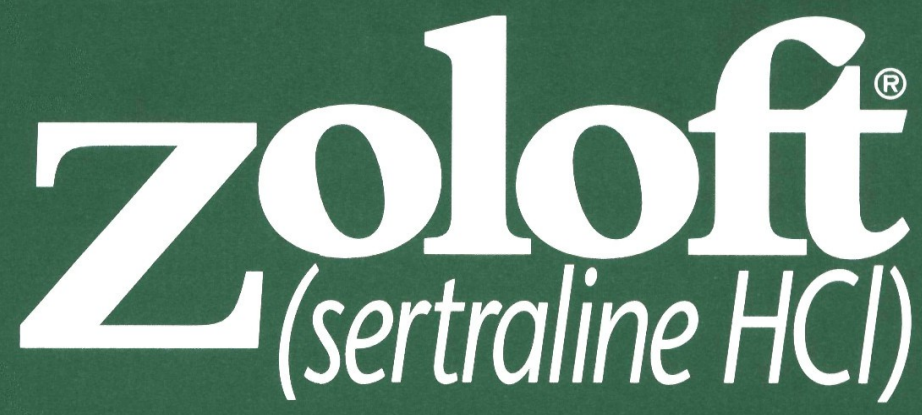




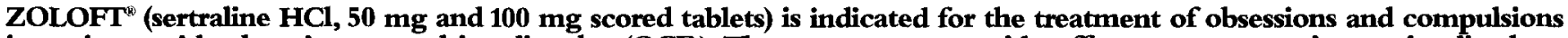
in patients with obsessive-compulsive disorder $(O C D)$. The most common side effects are nausea, insomnia, diarrhea, decreased libido, anorexia, dyspepsia, ejaculation failure (primarily ejaculatory delay), tremor, and increased sweating.

\section{BRIEF SUMMARY}

Zoloft

CONTRAINDICATIONS: Concomitant use in potients toking monoumine oxidase inhibitors (MA0ls) is controindicoted WARNINGS: Cases of serious sometimes fatal reactions have been reported in patients receiving ZOLOFT in combination with on MAOL. Therefore, it is recommended that ZOLOFT not be used in combination with an MAOL, or within 14 days of discontinuing treatment with an MAOI. Similarly, at least 14 days should be allowed after stopping ZOLOFT before starting an MAOI. PRECAUTIONS: General一During premarketing testing, hypomania or mania occurred in approximately $0.4 \%$ of 20 LOFT treated patients. Activation of mania/hypomania has also been reported in a small proportion of patients with Major Affective Disorder treated with other marketed antidepressant and antiobsessionol drugs. Weight Loss - Significant weight loss may be an undesirable result of treatment with sertraline for some patients, but an avernge, patients in controlled trials had minimni, it to 2 pound weight lass, versus smaller chonges on plocebo. Only rarely have sertraline patients been discontinued for weight loss. Seizure -70 LOFT has not been evaluated in patients with o seizure disorder. These patients were excluded from clinical studies during the product's premarket testing. No seizures were observed among approximately 3000 patients treated with ZOLOFT in the development program for depression. However, patients out of approximately 1800 exposed during the development program for obsessive compulsive disorder expesienced seizures, representing a crude incidence of $0.2 \%$. Three of these patients were oddescents, two with o seizure disorder and one with a family history of seizure disorder, none of whom were receiving anticonvulsont medication. Accordingly, like other antidepressant and antiobsessional drugs, 20LOFI should be introduced with care in patients with a seizure disorder. Suicide - The possibility of a suicide attempt is inherent in depression and may persist until significont remission occurs. Close supervision of thigh risk patients should accompany initiol drug therapy. Prescriptions for ZOLOFT should be written for the smallest quantity of toblets consistent with good potient monogement, in order to reduce the risk of overdose. Weok Uricosuric Effert-70L0FT is associated with o mean decrease in serum uric acid of opproximately $7 \%$. The clinical sitnificance of this week uricosuric effect is unknown, and there have been no reports of acute renol failure with ZOLOFT. Use in Patients with Concomitant lilness - Clinical experience with 20LOFT in patients with certain concomitant systemic illness is limited. Coution is advisable in using 20 LOFT in patients with disenses or conditions that could affect metabolism or hermodynamic responses. $7010 \mathrm{FT}$ has not been eveluated or used to any oppreciable extent in potients with a recent history of myocardiol infarction or unstabla heart disease. ZOLOFT is extensively metabolized by the liver. A lower or less frequent dose should be used in patients with cirrhosis. Until the pharmacokinetics of ZOLOFT hove been studied in patients with renal impairment and until adequate numbers of patients with severe renal impairment have been evaluated during chronic reotment with ZOLOFI, it should be used with coution in such patients. Interference with Cognitive and Motor Hyponatremia - Severol coses of hyponatremin have been reported. The hyponatienio oppeored to be reversible when ZOLOFT was discontinued. The majarity of these occurences have been in elderly individuals, some in potients taking diuretics or who were was discontinued. The majority of these occurences have been in elderly individuals, some in potients taking diuretics or who were
otherwise volume depleted. Platelet Function - There have been rare reports of altered platelet function and/or abnormal results from laborotory studies in patients taking ZOLOFT. While there hove been reports of abnormal bleeding or purpura in several patients toking 2010FT, it is unclear whether ZOLOFT had a cousative role. Information for Pafients: Patients shauld be told that although ZOLOFT has not been shown to impair the ability of normal subjects to perform tasks requiring complex motor and mental skills in laboratory experiments, drugs that act upon the central nervous system may affect some individuals adversely. Patients should be told that although 70 lOFi has not been shown in experiments with normal subjects to increase the mental ond motor skill impaiments caused by alcohol, the concomitant use of $70 \mathrm{LOFI}$ and alkohol in depressed or OCD patients is not advised. Patients should be told that while no odverse interaction of $20 \mathrm{LOFT}$ with over-the-counter (OTC) drug products is known to occur, the potential for interaction exists. Thus, the use of any OIC product should be initioted coutiousty according to the directions of use given for the 0TC product. Patients should be advised to notify their physicion if they become pregnant or intend to become pregnant during therapy. Pattients should be odvised to notify their physicion if they ore breast-feeding an infant. Laboratory Tesis: None Drug Interoctions: Polential Elfects of Coadministration of Drugs Highly Bound to Plasma Proteins Because sertraline is tightly bound to plasmo protein, the administration of $2010 \mathrm{FT}$ to a patient taking another drug which is tightly bound to protein $(e . g$. . Worfarin, digitoxin) may cause a shift in plasma concentrations potentially resulting in an adverse effect. Conversely, adverse effects may result from displacement of protein-bound 2OLOFT by other tightly bound drugs. Accordingly, prothrombin time should be carefully monitored when Z0LOFI theropy is initioted or stopped. Cimetidine - In o study assessing dis. position of $2010 F T(100 \mathrm{mg})$ on the second of 8 days of cimetidine administrotion ( $800 \mathrm{mg}$ daily), there were increases in $20 \mathrm{LOFT}$ mean AUC (50\%), Cmox (24\%) and half-life (26\%) compered to the placebo group. The clinical significance of these changes is unknown. CNS Active Drugs - in a study comporing the disposition of intravenously administered diazepom before and ofter 21 doys of dosing with either 20 LOFT ( 50 to $200 \mathrm{mg} /$ doy escolating dose) or plocebo, there was a $32 \%$ decrease relative to baseline in diazepam cleorance for the 20 LOFT group compared to a $19 \%$ decreose relative to baseline for the placebo group $(p<0.03)$. There wos a $23 \%$ increase in Tmax for desmethyldiazeparm in the Z0LOFT group compared to $020 \%$ decrease in the placebo group ( $<<0.03$ ). The clinical significance of these changes is unknown. In a placebo-controlled trial in normal volunteers, the administretion of two doses of $7020 \mathrm{FT}$ did not significontly alter steady-state lithium levels or the renal clearance of lithium. Nonetheless, at this time, it is recommended that plosma lithium levels be monitored following initiation of ZOLOFT therapy with appropriote odjustments to the lithium dose. The risk of using $70 \mathrm{LOFT}$ in combination with other CNS active drugs has not been systematically evaluated. Corsequently, caution is advised if the concomitront administration of ZOLOFI and such drugs is required. There is limited controiled experience regarding the optimal timing of switching from other ontidepresscnts to $2010 \mathrm{FI}$. Core and prudent medical judgment should be exerrised when switching, parficularly from long-acting agents. The duration of an oppropriate washout period which Drugs intervene before switching from one selective serotonin reuptcke inhibitor (SSRL) to another has not been established. (a) strated that sertraline coadministration did not increuse plasma concentrations of terfenadine or tarbamazepine. These data suggest that sertraline's extent of inhibition of P450 $3 A 4$ activity is not likely to be of clinical significance. Drugs Metabolized by P450 2D6 - Many antidepressonts, e.g. the SSRls, including sertraline, and most tricyclic antidepressants inhibit the biochemica activity of the drug metabolizing isozyme (vtochrome P450 206 (debrisoquin hydroxylase), and, thus, may increase the plasma concentrations of condrministered drugs that are metabolized by $\mathrm{P} 450206$. The drugs for which this potential interaction is of great est concern are those metabolized primarily by 206 and which have on norrow therapeutic index, e.g., the tricyclic antidepressants and the Type IC antiarthythmics propafenone ond flectinide. The extent to which this interaction is an important clinical problem depends on the extent of the inhibition of $\mathrm{P450} 206$ by the ontidepressont and the therapeutic index of the coadministered drug. There is variability among the antidepressants in the extent of clinically important 206 inhibition, and in fact sertraline at lower doses has a less prominent inhibitory effect on 206 than some others in the disss. Nevertheless, even sertraline has the potential for clinically impartant 206 intibition. Consequently, concomitant use of a drug metabolized by P450 206 with $20 L 0 F T$ may require lower doses thon usually prescribed for the other drug. Furthermore, whenever Z0LOFT is withdrawn from co-therapy, an increased dose of the coadministered drug may be required (see Iricyclic Antidepressants under PRECAUTIONS). Tricyelic Antidepressunts (TCAs) - The extent to which SSRI-ICA intercctions may pose clinical problems will depend on the
degree of inthibition and the pharmocokinetics of the SSRl involved. Nevertheless, coution is indicated in the condministration of degree of inhibition and the pharmocokinetics of the SSRl involved. Nevertheless, coution is indicated in the condministration of dose of TCA may need to be reduced, if a TCA is co-ndministered with ZOLOFT (see Drugs Metobolized by P450 206 under PRECALTIONS). Hypoglycemic Drugs - In a placebo-controlled trial in normal volunteers, administration of ZOLOFT for 22 days (including $200 \mathrm{mn}$ day tor the final 3 days) caused o statistically significant $16 \%$ decrease from baseline in the clearance of rolbutamide following on intravenous $1000 \mathrm{mg}$ dose. $2010 \mathrm{FT}$ administration did not noticeably change either the plasmo protein binding or the apparent volume of distribution of tolbutamide, suggesting that the deciecsed clewronce was due to a change in the rnetabolism of the drug. The clinical significance of this decrease in tolbutamide clearance is unknown. Atenolol - 20LOFT (100 mg) when administered to 10 healthy mole subjects had no effect on the beta-adrenergic blocking ability of atenolol. Digoxin - In a placebocontrolled trial in normal volunteers, administration of $20 \mathrm{LOFI}$ for 17 days (including $200 \mathrm{mg} /$ doy for the last 10 days) did not change serum digoxin levels or digoxin renol dearance. Microsomal Enzyme Induction - Preclinical studies have shown ZOLOFI to induce hepatic microsomal enzymes. In clinical studies ZOLOFI was shown to induce hepotic enzyrnes minimally as determined by a small (5\%) but statistically sigrificant decrease in antipyrine half-life following administration of $200 \mathrm{mg} / \mathrm{day}$ for 21 doys. This small change in antipyrine holf-life reflects a clinically insigniticant change in hepatic metabolism. Electroconvulsive Theropy - There ore no clinical studies establishing the risks or benefits of the combined use of electroconvulsive therapy (ECT) and 70 LOFT. Alcohol - Although $70 L \mathrm{LFT}$ did not potenticte the cognitive and psychomotor effects of alcohol in experiments with normal subjects, the concomitant use of 70LOFF and alcohol in depressed or OCD patients is not recomrnended. Carcinogenesis, Mufagenesis, Impairment of Fertility: Lifetime carcinogenicity studies were carried out in (D-1 mice and Long-Evans rats at
doses up to $40 \mathrm{mg} / \mathrm{kg} /$ day. These doses correspond to 1 times (mice) and 2 times (rots) the maximum recommended human dose
(MPHD) on a mg/ $\mathrm{m}^{2}$ basis. There was a dose-related increase of liver adenomas in male mice receiving sertroline at $10-40 \mathrm{mg} / \mathrm{kg}$ $\left(0.25-1.0\right.$ times the $M R H D$ on a $\mathrm{mg} / \mathrm{m}^{2}$ bosis). No increase was seen in female mice or in rats of either sex receiving the same treatments, nor was there on increase in hepatocellular carcinomas. Liver adenomas hove a variable rate of spontaneous occurrence rats receiving sertraline at $40 \mathrm{mg} / \mathrm{kg}$ (2 times the MRHD on a mg/m $/ \mathrm{m}^{2}$ basis); this wos not accompanied by thyroid hyperplasia. While there was an increase in uterine adenocorcinomas in rats receiving sertraline of $10-40 \mathrm{mg} / \mathrm{kg}(0.5-2.0$ times the MRHD on a $\mathrm{mg} / \mathrm{m}^{2}$ bosis) compared to placebo controls, this effect wos not clearly drug related. Sertroline had no genotoxic effects, with or without metabolic activation, based on the following ussoys: bacterial mutation assay, rnouse lymphoma mutation assay; and tests for cytogenetic oberrations in vivo in mouse bone marrow and in vitro in hurrian lymphocytes. A decrease in fertility wos seen in one of two rat studies at a dose of $80 \mathrm{mg} / \mathrm{kg}$ (4 times the moximum human dose on o $\mathrm{mg} / \mathrm{m}^{2}$ basis). Pregnancy - Pregnancy
Category C: Reproduction studies hove been performed in rats ond rabbits of doses up to $80 \mathrm{mg} / \mathrm{kg} /$ day and $40 \mathrm{mg} / \mathrm{kg} / \mathrm{day}$, respectively. These doses correspond to approximately 4 times the maximum recommended human dose (MRHD) on o $\mathrm{mig} / \mathrm{m}^{2}$ bosis. There was no evidence of terotogenenicity of any dose level. When pregnont rots ond robbits were given sertraline during the period of organogenesis, deloyed ossification was observed in fetuses at doses of $10 \mathrm{mg} / \mathrm{kg} 10.5$ times the MRHD on a mg/m basis) in rats and $40 \mathrm{mg} / \mathrm{kg}$ ( 4 times the MRHD on a mg/m² basis) in rabbits. When femole rats received sertraline during the last third of gestation and throughout loctation, there wos an increase in the number of stillborn pups and in the number of pups dying
during the first 4 doys after birth. Pup body weights were olso decreased during the first fous doys ofter birth. These effects ocured at a dase of $20 \mathrm{mg} / \mathrm{kg}$ (1 times the MRHD on a mg/m² basis). The no effert dose for rat pup rnortality was $10 \mathrm{mg} / \mathrm{kg}(0.5$ times the MRHD on a $\mathrm{mg} / \mathrm{m}^{2}$ bosis). The decrense in pup survival was shown to be due to in utero exposure to sertraline. The clinical significance of these effects is unknown. There ore no adequate and well-controlled studies in pregnant women. 20LOFI should be used during pregnancy only if the potential benefit justifies the potential risk to the fetus. Labor ond Delivery - The effect of $70 \mathrm{LOFT}$ on lobor and delivery in humans is unknown. Nursing Mothers - It is not known whether, ond if so in what amount, sertraline or its metabolites are excreted in human milk. Becouse meny diugs are excreted in human milk, coution should be exercised when 20LOFT is administered to a nursing woman. Pediatric Use - Safety and effectiveness in children have not been established. tions in the elderly was similar to that in younger patients. ADVERSE REACIIONS Commonly Observed: Among patients treated with $Z 010$ OFT in placebo-controlled premmorketing studies, the most commonly observed adverse events associnted with the treated with ZOLOFT in placebo-controlled premorketing studies, the most commonly observed adverse events associnted with the
use of $7010 \mathrm{OFT}$ and not seen ot an equivalent incidence among placebo-treated petients were: gastrointestinal comploints, induding nauseo $(26 \%$ vs $12 \%)$, diartheo/loose stools $(18 \%$ vs $9 \%$ ) and dyspepsia $(6 \%$ vs $3 \%)$; tremor $(11 \%$ vs $3 \%)$; dizziness $(12 \%$ vs $7 \%)$; insomnia $(16 \%$ vs $9 \%)$; somnolence $(13 \%$ vs $6 \%$ ); increased sweating $(8 \%$ vs $3 \%) ;$; dry mouth $(16 \%$ vs $9 \%)$; and male sexval dysfunction ( $16 \%$ vs $2 \%$ ), primarily ejcculatory delay. In plocebo-controlled clinical trials for $\mathrm{OCD}$, adverse events observed at an incidence of at least $5 \%$ for $20 \mathrm{LOFT}$ and at an incidence that wos twice or more the incidence among placebo-treated patients includ-

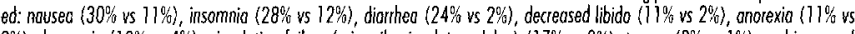
$2 \%$ ), dyspepsia $(10 \%$ vs $4 \%)$, ejaculation failure (primarily ejoculatory delay) $(17 \%$ vs $2 \%$, tremor $(8 \%$ vs $1 \%)$, and increased 5weating (6\% vs $1 \%$ ). Associated with Discontinuation of Treatment: Fifteen percent of 2710 patients who received
20LOFT in premarketing multiple dose clinisal trials discontinued treatment due to an adverse event. The more common events (reported by at least $1 \%$ of subjects) associoted with discontinuation induded agitation, insomnia, male sexual dysfunction (primurin ejoculatory delay), somnolence, dizziness, headache, tremor, onorexia, diorrheo/10ose stools, nausea, and fotigue. In placebocontrolled dlinical trials for OCD, 10\% of patients treated with $20 \mathrm{LOFT}$ discontinued treatment due to an adverse event. The more common events were nausea, insomnia, and diarhea. Other Events Observed During the Premarketing Evaluation of ZOLOFT: During its premarketing assessment, multiple doses of ZOLOFT were administered to approximotely 2700 subiects. Events are further categorized by body system and listed in order of decreasing frequency according to the following definitions: frequent adverse events are those occurring on one or more occasions in at least $7 / 700$ patients (only those not already listed in the (1000 results from placebo-controlled triols appear in this listing); infrequent adverse events ure those occurring in $1 / 100$ to described in the PRECAUTIONS section. Autonomic Nervous System Disorders - Infrequent: flushing, mydriasis, increused saliva, cold clommy skin; Rore: pallor. Cordiovascular - Infrequent: postural dizziness, hypertension, hypotension, postural hypotension, edema, dependent ederna, periorbital edema, peripheral ederna, peripheral ischemia, syncope, tachycardia; Rore: pre-
cordial chest pain, substernal chest poin, aggravated hypertension, myocordial infarction, voricose veins. Central and Periphercordial chest pain, substernal chest pain, oggravated hypertension, myocordial inforction, voricose veins. Central and Peripher-
al Nervous System Disorders - Frequent: confusion; Infrequent: ataxia, cbnormol coordination, obnormal gait, hyperesthesia, hyperkinesia, hypokinesio, migraine, nystagmus, vertigo; Rare: local onesthesia, como, convulsions, dyskinesia, dysphonia, hyporeflexin, hypotonia, ptosis. Disorders of Skin and Appendages - Infrequent: acne, alopecin, pruritus, erythematous

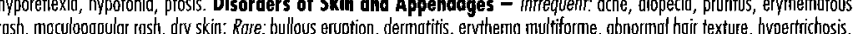

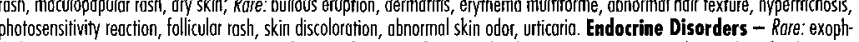
halmos, gynecomastio. Gastrointestinal Disorders - Infrequent: dysphagia, eructotion; Rare: diverticulitis, fecal incontinence, gastritis, gastroenteritis, glossitis, gum hyperplasio, hemorrhoids, hiccup, melena, hemorrhogic peptic ulcer, proctitis, stomeralized edema, rigors, weight decrease, weight incrense; Rore: enlorged abdomen, halitosis, ofitis media, aphthous stomatitis. Hematopoietic and Lymphatic - Infrequent: lymphodenopathy, purpuro; Rose: onemio, anterior chamber eye hemorrhoge. Metabolic and Nutritional Disorders - Rare: dehydration, hypercholesterolemia, hypoglycemia. Musculoskeletal SysMetabolic and Nutritional Disorders - Rare: dehydration, hypercholesterolemia, hypoglycemia. Musculoskeletal Sys-
tem Disorders - Infrequent: arthrolgia, arthrosis, dystonia, muscle cramps, muscle weakness; Rare: hemia. Psychiatric Disorders-Infrequent: abnormal dreams, aggressive reaction, amnesia, apathy, delusion, depersonalization, depression, aggravated depression, emotional lability, euphoria, hallucination, neurosis, peranoid reaction, suicide ideation and attempt, teeth-grinding, abnormal thinking; Rore: hysteria, somnambulism, withdrowol syndrome. Reproductive - Infrequent: dysmenorrhea (2), intermenstrual bleeding (2); Rare: amenorntea (2), balanoposthitis (1), breast enlargement (2), female breost poin (2), leukorrheo (2), menorrhagio (2) atrophic vagiritis (2). (1) -\% based on male subjects only: 1005; (2) - \% based on femole subjects only: 1705. Respiratory System Disorders - Infrequent: bronchospasm, coughing, dyspnea, epistaxis; Rave: brodypnea, hyperventilation, sinusitis, stridor. Special Senses - Infrequent: abnormal accommodation, conjunctivitis, diplopia, earache, eye pain, xerophthalmia; Rare: abnormal lacrimation, photophobia, visual field defect. Urinary System Disorders - Infrequent: dysuria, foce edema, nocturia, polyuria, urinary incontinence; Rare: oliguria, renal poin, urinary retention. Laboratory Tests: In man, symptomatic elevations in serum transaminoses (SGOT [or AST] and SGPT [or ALI]) have been reported infrequently (approxiweeks of drug treatment and promptly diminished upon drug discontinuation. ZOLOFT therapy wos ossocioted with small mean increases in total cholesterol (approximately $3 \%$ ) and triglycerides (approximately $5 \%$ ), and a small mean decrease in serum uric acid (opproximately $7 \%$ ) of no apparent clinical importance. The safety profile observed in OCD patients treated with Z0LOFT is simlar to the sofety profile in depressed potients. DRUG ABUSE AND DEPENDENCE Controlled Substance Class - 7OLOFT not a controlled substance. Physical and Psychological Dependence - 20LOFT has not been systematically studied, in with ZOLOFT did not reveal any tendency for a withdrawal syndrome or any drug-seeking behovior. As with any new CNS active drug, hysicians should carefully evoluate patients for history of drug abuse and follow such patients closely observing them for signs of physicians should carefully evaluate potients for histary of drug abuse and follow such patients dlosely, observing them for signs of
zOLOFT misuse or abuse (e.g., development of tolercence, incrementation of dose, drug-seeking behovior). OVERDOSAGE Human Experience - As of November, 1992, there were 79 reports of non-fatal ocute overdoses involving ZOLOFT, of which 28 were overdoses of 70 LOFT alone and the remainder involved a combination of other drugs and/or akohol in addition to ZOLOFT. In those coses of overdose involving only ZOLOFT, the reported doses ranged from $500 \mathrm{mg}$ to $6000 \mathrm{mg}$. In a subset of 18 of these patients in whom ZOLOFT blood levels were determined, plosma concentrations ranged from $<5 \mathrm{ng} / \mathrm{mL}$ to $554 \mathrm{ng} / \mathrm{mL}$. Symptoms of overdose with $70 \mathrm{LOFT}$ alone induded samnolence, nousec, vomiting, tachycardia, $\mathrm{ECG}$ changes, anxiety and diloted puils. Treatnent wos primarily supportive and included monitoring and use of activoted chascoal, gustric lavoge or cathartics and hydration. Although there were no reports of denth when ZOLOFT was taken alone, there were 4 deaths involving overdoses of ZOLOFT in combination with other drugs and/or olcohol. Therefore, any overdosage should be treated aggressively. Manogement of Overwith sorbitol, moy be as or more effective than emesis or lavage, and should be considered in treating overdose. Cordiac and vital signs monitoring is recommended along with gernerol symptomatic and supportive measures. There are no sperific ontidates for ZOLOFT. Due to the large volume of distribution of ZOLOFT, forced diuresis, diolysis, hemoperfusion, and exchange transfusion are unlikely to be of benefit. In managing overdosage, consider the possibility of multiple drug involvement. The physician should consider contocting a poison control center on the treatment of ony overdose. 